\title{
Oferta natural y estado actual de conservación de las poblaciones de Damagua (Poulsenia armata), en los bosques nativos de las comunidades indígenas del río Amporá, Alto Baudó, Chocó
}

\author{
Natural offer and conservation state of populations Poulsenia armata \\ in native forest of indigenous communities of Amporá river, Alto Baudó, \\ Chocó
}

\author{
Giovanny Ramírez-Morenoํㅜ, Zoraida Quezada Martinez², Lady Vargas Porras ${ }^{3}$, \\ Zulmary Valoyes Cardozo 4
}

\section{Resumen}

Se determinó la oferta de la Damagua (Poulsenia armata) y su respectiva demanda en los mercados locales, tomando como base el estado actual de poblaciones naturales en tres comunidades indígenas del río Amporá. En cada localidad (Agua Clara, Bella Luz y Londoño) se establecieron 20 transectos de $5 \mathrm{~m}$ x $20 \mathrm{~m}\left(2.000 \mathrm{~m}^{2}\right)$, en los cuales se identificaron individuos, se estableció su categoría de tamaño y su morfología teniendo en cuenta una combinación entre diámetro y altura, además su abundancia y estado vegetativo y reproductivo. Se obtuvo la oferta del recurso con la densidad de individuos juveniles calculado como número de árboles, sabanas y metros cuadrados de fibra por área muestreada. Se determinó la demanda mediante entrevistas con los artesanos. Se encontró que el mayor porcentaje de individuos corresponde al estado juvenil (62\%), seguido de los infantes (25\%) y por último los adultos (13\%). Se identificaron tres categorías de desarrolloy cuatro clases diamétricas, a partir de las cuales se determinó que la oferta total de fibra equivale a 229 árboles por 1.5 ha. Los resultados mostraron la superioridad de la oferta frente a la demanda y se convierten en elementos diagnósticos del buen estado de las poblaciones de Damagua en estas comunidades y al mismo tiempo demuestran que las técnicas de aprovechamiento empleadas por los indígenas constituyen un elemento facilitador para el diseño de herramientas de manejo sostenible de los recursos del bosque.

Palabras clave: Damagua; Poulsenia armata; Productos no maderables del bosque; Análisis poblacional de especies vegetales.

\begin{abstract}
There decided the offer of the Damagua (Poulsenia armata) and his respective demand on the local markets, taking as a base the current condition of natural populations in three communities indigenous to the Amporá river. In every locality (Agua Clara, Bella Luz and Londoño) established 20 transectos of $5 \mathrm{~m}$ $\times 20 \mathrm{~m}\left(2.000 \mathrm{~m}^{2}\right)$, in which individuals identified, there was established his category of size and his morphology having in it counts a combination between diameter and height; in addition his abundance and vegetative and reproductive condition. The offer of the resource was obtained by the density of juvenile individuals calculated as number of trees, sheets and square meters of fiber by sampled area. The demand decided by means of interviews with the craftsmen.
\end{abstract}

1 Biólogo, Magíster en Ciencias Biológicas; Investigador principal Componente Ecosistémico, Instituto de Investigaciones Ambientales del Pacífico, Quidbó, Colombia. e-mail: gramirezm3@hotmail.com

2 Ingeniera Ambiental, Especialista en Ordenamiento y Gestión Integral de Cuencas Hidrográficas, Residente ambiental, Metro-corredores. Quibdó, Colombia. e-mail: zoraidaquesada@hotmail.com

3 Ingeniera Ambiental, Especialista en Manejo Integrado de Recursos Hídricos, Investigadora Instituto de Investigaciones Ambientales del Pacífico. Quidbó, Colombia.

e-mail: lvargas@iiap.org.co

4 Bióloga, Especialista en Administración de Recursos Naturales, Investigadora Instituto de Investigaciones Ambientales del Pacífico. Quidbó, Colombia. e-mail:zulmaryvaloyes@hotmail.es Recibido: 25 de enero de 2012 Aceptado: 30 de abril de 2012 
Conservación, oferta natural y estado actual de Poulsenia armata. G. Ramírez-Moreno et al.

One thought that the major percentage of individuals corresponds to the juvenile condition (62\%), followed by the infants (25\%) and finally the adults (13\%). There were identified three categories of development and four classes diamétricas, from which one determined that the total offer of fiber is equivalent to 229 trees for 1.5 ha. The results showed the superiority of the offer opposite to the demand and they turn into diagnostic elements of the good condition of the populations of Damagua into these communities; at the same time they demonstrate that the technologies of utilization used by the aborigens constitute an element facilitator for the design of tools of sustainable managing of the resources of the forest.

Keywords: Damagua; Poulsenia armata; Non-timber forest products; Population analysis of vegetable species.

\section{Introducción}

Entre los usos más importantes dados a las materias primas extraídas del bosque en el Chocó está el artesanal, que involucra básicamente semillas, colorantes y fibras siendo las fibras las que actualmente tienen un mayor mercado regional y una gran proyección hacia el nacional e internacional.

Esta oferta de productos se derivan de la flora chocoana, la cual ha sido reconocida a nivel mundial por sus altos atributos de riqueza, diversidad biológica y ecosistémica; se estima que el departamento del Chocó posee más de 6000 especies de plantas. Los vacíos de información básica sobre taxonomía, etnobotánica, ecología y distribución de la flora, no ha permitido el desarrollo de manuscritos donde se precisen estas cifras, pero los múltiples, constantes y recientes descubrimientos en este territorio hacen creer que son ciertas las estimaciones de Forero, Gentry (1979) y Gentry (1981) sobre la ocurrencia de 7000-8000 especies de plantas. Esta alta riqueza natural ha sido sin duda alguna utilizada ampliamente por comunidades nativas que derivan su sustento del medio. Se estima que entre $20 \%$ y $30 \%$ de las plantas del Chocó son usadas o tienen un uso potencial. Análisis realizados por Pino et al. (2001), sobre los especímenes depositados en el herbario de la Universidad Tecnológica del Chocó arrojan que de las 2100 especies, 672 (32\%) se encuentra distribuidas en alguna de las siguientes categorías de uso regional: artesanales, alimenticias, medicinales, construcción y ornamentales. Investigadores como Macía et al. (2001), relacionan la diversidad de usos con la diversidad de especies.

Lo anterior confirma que la alta diversidad vegetal de los bosques del Chocó Biogeográfico, ha representado y asegurado históricamente una alta oferta de recursos (maderables y no maderables) para las poblaciones humanas que aquí habitan. Esta fuerte relación se ha mantenido gracias a que la extraordinaria oferta ha estado íntimamente ligada a una baja demanda que permite que pequeñas comunidades afrodescendientes o indígenas vivan de este potencial sin causar efectos estructurales, porque las técnicas de aprovechamiento de los recursos del bosque, se hacen con tecnologías propias que permiten la resiliencia y el equilibro de la biodiversidad de esta importante región.

En esta investigación se hace una contribución al conocimiento de la oferta de las poblaciones natural de la Damagua (Poulsenia armata) y su respectiva demanda en los mercados locales, que hacen a esta especie la productora a nivel regional de fibra más importante para la elaboración de artesanías; esta situación convierte a sus poblaciones naturales y a los bosques que las contienen en los únicos sitios para acceder a este importante recurso no maderable, lo que prende la alarma de la sostenibilidad en el tiempo del aprovechamiento del recurso. Para llegar a esta contribución se conformó una alianza entre el IIAP, DAMAGUARTE y las comunidades indígenas del río Amporá, la cual permitió conocer los estados poblacionales, ofertas y demandas del recurso, elementos básicos que servirán como base científica para el desarrollo de posteriores herramientas de manejo sostenible participativo de tan importante recurso.

Área de estudio. El Alto Baudó, que está localizado en el departamento del Chocó, a $80 \mathrm{~km}$ de su capital. Este municipio se encuentra ubicado a los 5'31'33" de latitud norte y a los 76 59'44"de longitud oeste; su casco urbano alcanza una altura de $50 \mathrm{msnm}$ y tiene una extensión de $1.532 \mathrm{~km}^{2}$, que representa aproximadamente $3.2 \%$ de la superficie departamental. Su cabecera Pie de Pató, está localizada en la margen izquierda del río Baudó. Limita por el norte con los municipios de Nuquí, Bojayá y Bahía Solano; por el sur con los municipios de Medio Baudó y Bajo Baudó; por el oriente con los municipios de Quibdó, Río Quito, Cantón del San Pablo y por el este con los municipios de Bajo Baudó y Nuquí.

Resguardo Agua Clara y Bella Luz. Las comunidades indígenas Embera de Agua Clara y Bella Luz, se encuentran ubicadas en ambas márgenes de la parte alta del río Amporá, en jurisdicción del municipio de Alto Baudó, departamento del Chocó. Su posición geográfica corresponde a los $5^{\circ} 20^{\prime} \mathrm{de}$ latitud norte y $77^{\circ} 2^{\prime}$ de longitud oeste. Para llegar al resguardo indígena de Bella Luz y Agua Clara, se debe hacer el recorrido por vía fluvial aguas abajo del río Baudó, desde la cabecera municipal del Alto Baudó hasta donde se conecta el río Baudó con el río Amporá, donde se continúa el recorrido aguas arriba por el río Amporá. La duración del recorrido depende del nivel del agua que tenga este último; si este es alto, el recorrido tiene una duración de tres horas y si el nivel del agua es bajo, el recorrido tiene una duración de seis a siete horas.

Resguardo Dominico Indio y Londoño. Las comunida- 
Bioetnia Volumen 9 No 1 (enero-junio), 2012

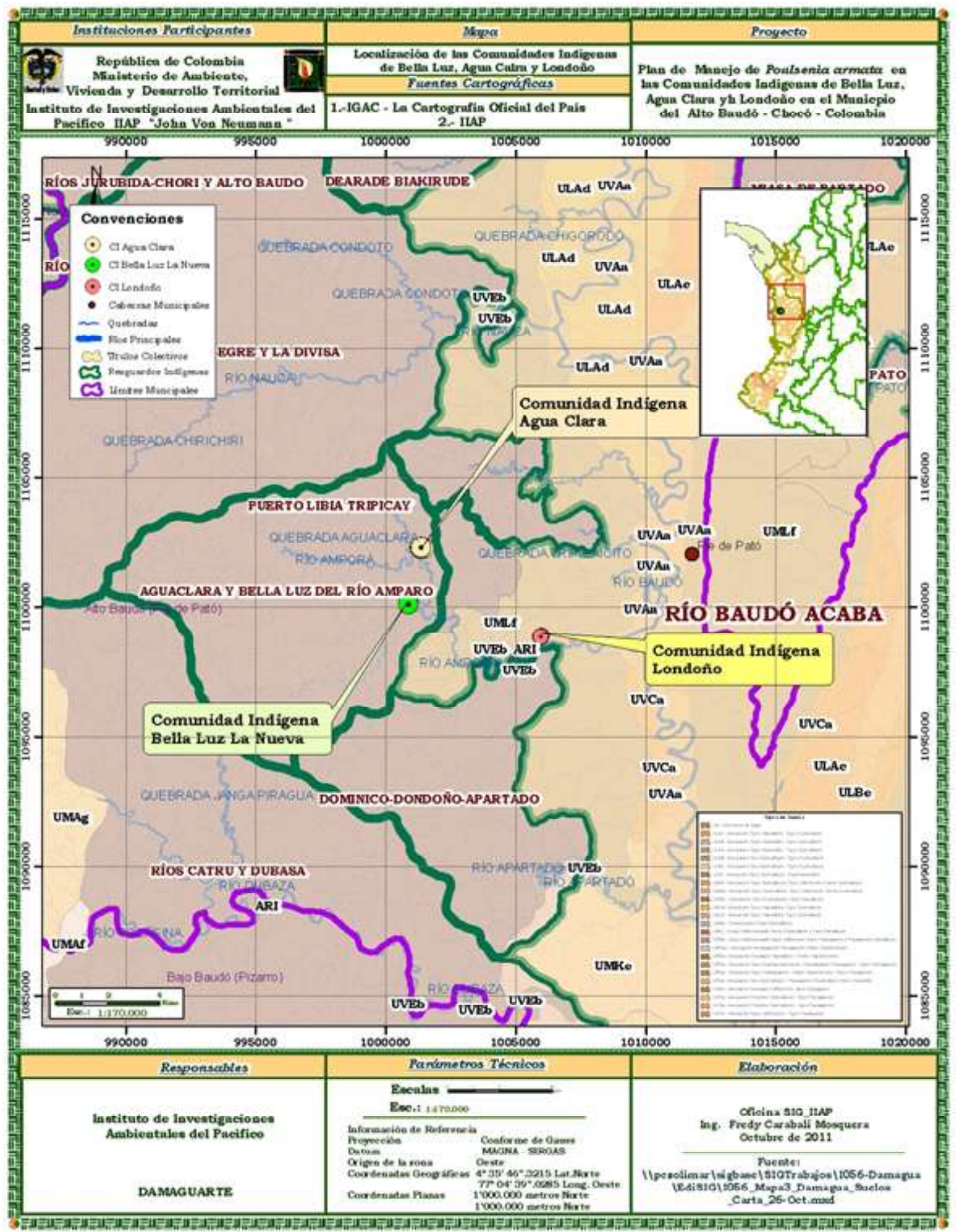

Figura 1. Mapa de los resguardos indígenas

des indígenas Embera de los ríos Dominico y Londoño hacen parte de la Hoya Hidrográfica del río Amporá y en el curso del río Apartadó, cuyas aguas desembocan en el Baudó, en jurisdicción del municipio del Alto Baudó (Figura 1). El área 
del resguardo es de 6.610 hectáreas aproximadamente. La comunidad indígena Embera del río Londoño, está compuesta por 31 personas nucleadas en 7 familias, con un promedio de 4.4 personas por familia (CODECHOCO, 2010). Para llegar al resguardo indígena Dominico Indio y Londoño, se debe hacer el recorrido por vía fluvial aguas abajo del río Baudó, desde la cabecera municipal del Alto Baudó hasta donde se conecta el río Baudó con el río Amporá, donde se continúa el recorrido aguas arriba por el río Amporá; la duración del recorrido es de media hora.

\section{Métodos}

Concertación con la comunidad. Se realizó esta etapa con varios propósitos: en primer lugar informar a los habitantes de cada localidad de estudio sobre las pretensiones del proyecto y sus resultados esperados, la metodología propuesta para desarrollarlo y los impactos que eventualmente se generarían con la aplicación de la información aportada; en segundo lugar, para lograr acuerdos en cuanto a los mecanismos de participación de la comunidad, definir los sitios precisos de muestreo y las rutas y costos necesarios para su acceso y avanzar en la identificación del personal de la zona que podría vincularse con el proyecto (Figura 2).
Fase de campo. Los muestreo se realizaron en transectos que se distribuyeron de manera sistemática a lo largo de los diferentes microambientes (pendientes, planicies y bosques de influencia hídrica), teniendo en cuenta que la distancia mínima entre transectos fuera de $20 \mathrm{~m}$, que no se solaparan, ni existiera en su interior zonas muy alteradas como caminos amplios o claros de bosque. En cada una de las localidades (Agua Clara, Bella Luz y Londoño) se establecieron 20 transectos de $5 \mathrm{~m} \mathrm{x} 20 \mathrm{~m}\left(2.000 \mathrm{~m}^{2}\right)$, para un área de 0.2 ha. El área total cubierta por todos los transectos en las tres localidades fue de 0.6 ha. La delimitación de los transectos se hizo utilizando una cuerda como eje central y dos varas de madera de $2.5 \mathrm{~m}$ a lado y lado de la cuerda. En cada transecto se registraron y midieron todos los individuos de Damagua, incluyendo todas las formas de crecimiento (plántulas, infantes, juveniles y adultos).

Se hicieron colecciones completas de la planta en los transectos y colecciones adicionales en los sectores aledaños a los transectos. Se recolectaron cinco ejemplares, preferiblemente en estado reproductivo, los que sirvieron de base para asegurar la adecuada identificación de la especie. La información se complementó con registros fotográficos. Toda la información de la especie por localidad (nombre vulgar y científico, usos, descripciones, entre otras), fue consignada

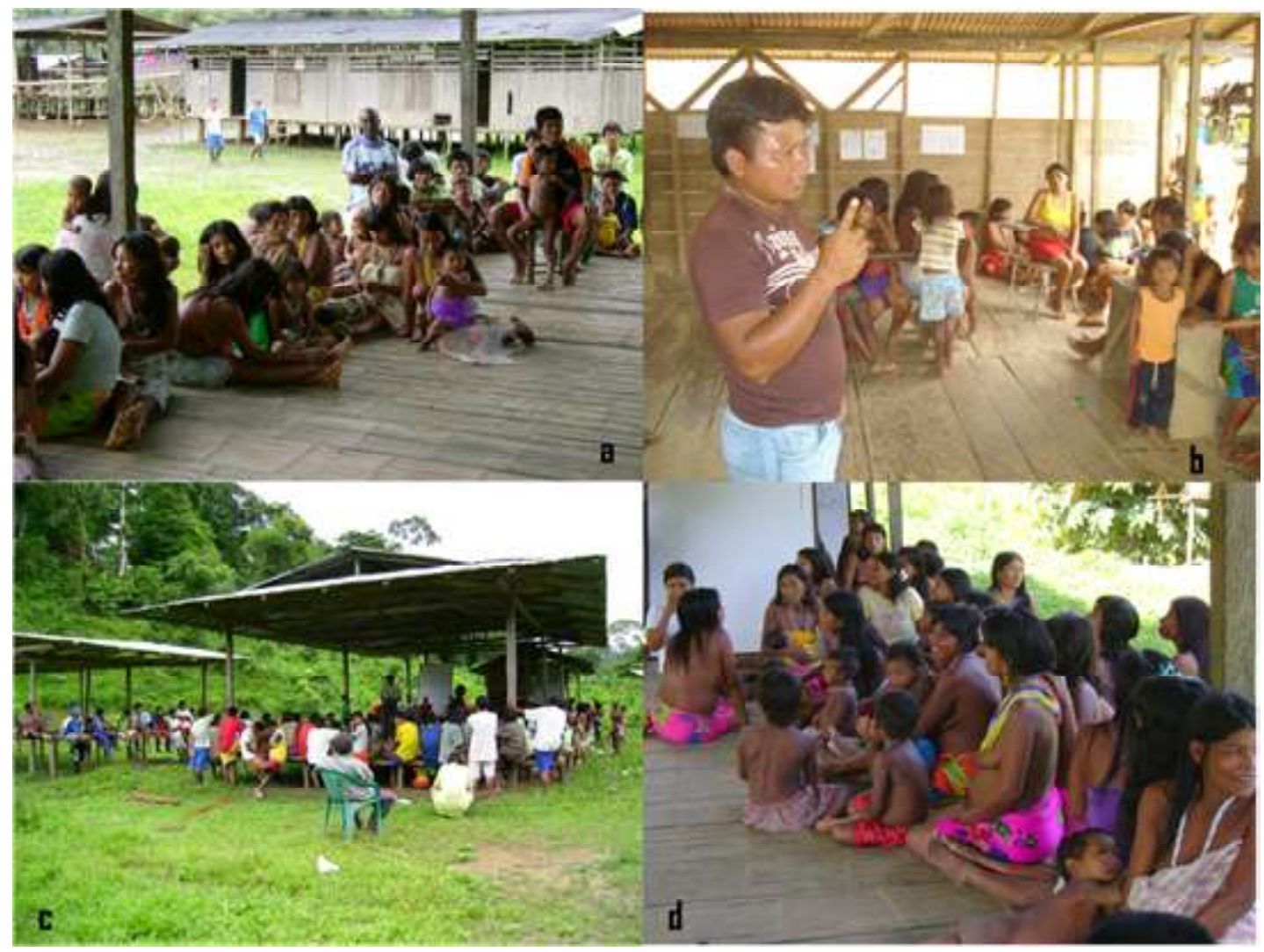

Figura 2. Concertación comunitaria. 


\section{Bioetnia Volumen 9 No 1 (enero-junio), 2012}

en una hoja de cálculo de Excel 2007.

Para establecer las alturas de los individuos de Damagua se utilizó un clinómetro y por medio de estas mediciones se pudieron establecer los siguientes rangos de altura $1=0.5-5$ $\mathrm{m} ; 2=6-15 \mathrm{~m} ; 3=16-30 \mathrm{~m}$.

Para documentar las categorías de tamaños y la morfología de la especie se tuvo en cuenta una combinación entre diámetro y altura; además, las estructuras vegetativas y reproductivas (forma, color, longitud y estructura de raíz, tallo, hoja, flores y frutos). Lo anterior permitió definir las siguientes categorías: infantes, individuos con un estado de desarrollo más avanzado que las plántulas con un diámetro entre 1 a $10 \mathrm{~cm}$ y una altura de 0.5 a $5 \mathrm{~m}$; juveniles, con un estado de desarrollo avanzado, donde se identifican claramente las características vegetativas particulares de la especie, pero sin estructuras reproductivas evidentes o inferidas, con una altura entre 5.1 y $15 \mathrm{~m}$ y un diámetro que va de 10.1 a $20.5 \mathrm{~m}$; y adultos, individuos que presentan alguna evidencia reproductiva como cicatrices, brácteas, frutos o semillas viejas, con alturas mayores de $15 \mathrm{~m}$ y diámetro mayor de 20 $\mathrm{m})$. Las abundancias por categorías de tamaños se calcularon a través de las sumatoria del número de individuos de la categoría por transecto, luego por localidad y a nivel general de la zona.

Usos de la Damagua por parte de los pobladores asentados en la zona de influencia del proyecto. En cada localidad se registró la información suministrada por cinco guías de campo y tres conocedores tradicionales (16 personas), todos hombres mayores de 45 años, nativos de las localidades, reconocidos por los habitantes y resguardos indígenas como conocedores de los bosques. La información etnobotánica se obtuvo directamente en campo durante el trabajo en los transectos. Las preguntas acerca del uso de la Damagua fueron abiertas, dejando que los entrevistados mencionaran los usos conocidos, los nombres comunes y la parte aprovechada.

Fase de laboratorio. El material colectado se trasladó al Laboratorio de Botánica y Ecología de la UTCh, una vez descrito y secado, se confrontó con claves taxonómicas especializadas y se comparó con algunos herbarios virtuales como el New York Botanicals Garden (NY), Neotropical Herbarium Specimens (http://fm.fieldmuseum.org/vrrc), entre otros sitios disponibles; la especie fue descrita teniendo en cuenta la metodología clásica utilizada en taxonomía.

Para establecer la oferta del recurso se utilizó la densidad de individuos juveniles obtenida en campo, porque esta clase de tamaño constituye por sus características, la población aprovechada por las comunidades. A partir de esto se calculó la oferta en términos de número de árboles por el total de área muestreada, número de sábanas por área y cantidad de metros cuadrados de fibra obtenidos por área muestreada. Lo anterior teniendo en cuenta las relaciones de cantidad proporcio- nadas por los extractores, las cuales indican que de cada árbol de Damagua se obtienen cerca de 5 sábanas que miden cada una $0.80 \mathrm{~m}$ de ancho por $1.20 \mathrm{~m}$ de largo.

Para el cálculo de la demanda, se estableció mediante entrevista con los artesanos, quienes proporcionaron datos de la frecuencia anual de compra de fibra y la cantidad por vez. Dicha cantidad estuvo expresada en bultos que se convirtieron a árboles teniendo en cuenta las proporciones antes mencionadas.

\section{Resultados y discusión}

En 60 transectos $(5 \times 20 \mathrm{~m})$ correspondientes a un área de 0.6 ha de tres comunidades indígenas del río Amporá, cuenca alta del río Baudó, se registraron 386 individuos de la especie Poulsenia armata, presentando la comunidad de Agua Clara $142(37 \%)$ de los individuos que ocurren en los muestreos, seguida de Bella Luz con 126 (33\%) individuos y de Londoño con $30 \% 118(30 \%)$ de individuos restante.

Distribución por clases de tamaño de la población de Damagua. Los datos generales del conteo de individuos contenidos en los transectos, permitieron distribuir los 386 individuos de la población de Damagua, en los estados de desarrollo plántulas (infantes), juveniles y adultos de la siguiente manera: el mayor porcentaje de individuos se encontró en el estado juvenil (62\%), seguido de los infantes (25\%) y por último los adultos (13\%).

Las estructuras poblacionales por localidades extractoras de la fibra, permiten observar el fuerte domino de las categorías de tamaño juveniles y plántulas, lo que podría estar representando una población en buen estado, porque en un modelo de distribución normal de poblaciones sanas ( Jinvertida) estas dos categorías deben ser dominantes, aunque el orden de dominancia (más infantes que juveniles) no se sigue en esta distribución, es muy posible que aquí no se haya observado por el estado fenológico reproductivo de los individuos maduros. En el momento de los muestreos no fue posible observar ningún evento reproductivo lo que sugiere que en las poblaciones el proceso de reclutamiento de plántula a juvenil estuviese avanzado (Figura 3 ).

Clases diamétricas. Los individuos de Damagua encontrados en estos levantamientos se agrupan en las clases 1, 2, 3 y 4 y se distribuyeron así: 59 (43\%) para la clase 2, 29(42\%) para las clase 1 , con un fuerte predominio de individuos de diámetros de $10 \mathrm{~cm}$ y el restante $15 \%$ distribuidos en las clases 3 y 4 con $8(10 \%)$ y $3(5 \%)$ respectivamente. Esta distribución muestra una clara oferta del recurso para los diámetros entre 10 y $21 \mathrm{~cm}$.

Oferta y demanda del recurso. Para evaluar el aprovechamiento sostenible de plantas que son extraídas de los bosques, es necesario determinar la oferta natural del recurso (Cunningham 2001, Wong et al. 2001), lo cual se realiza 


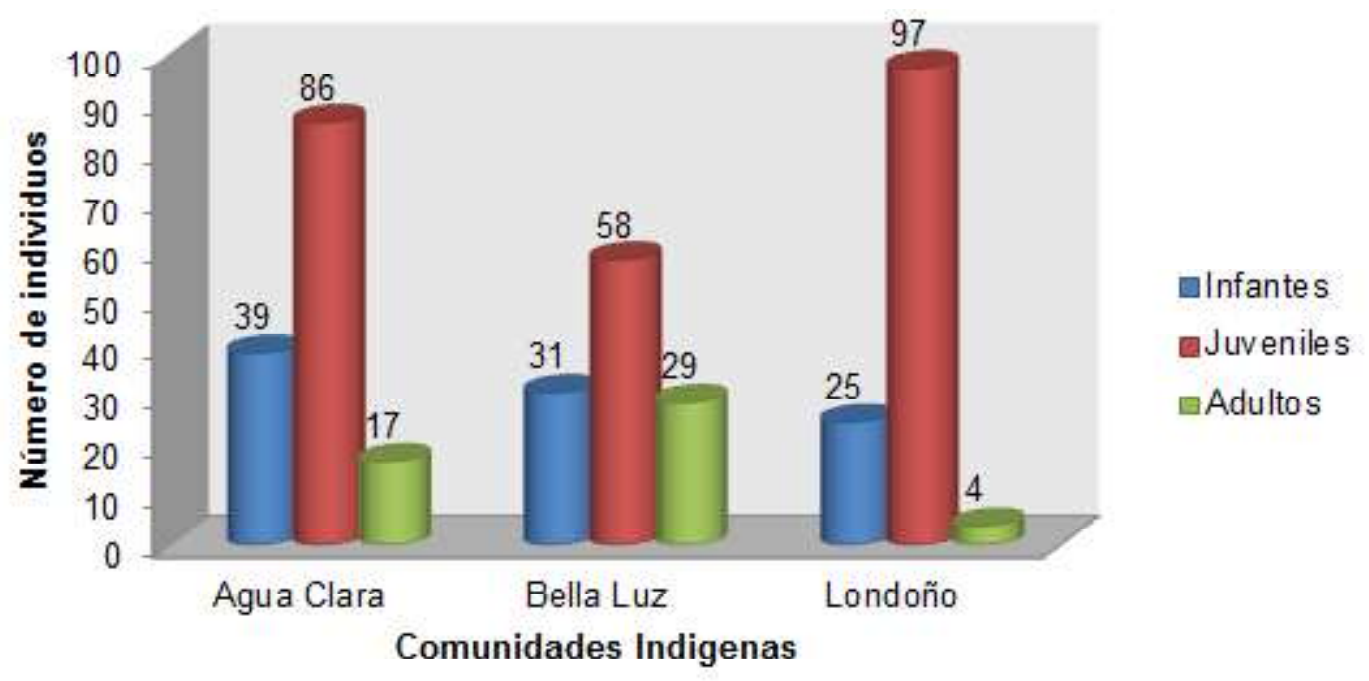

Figura 3. Distribución de las clases de tamaño por localidades

teniendo en cuenta tres aspectos: el primero es el volumen del recurso, que se mide a partir de la abundancia de especie y la parte aprovechada; el segundo es la productividad del recurso, que se puede estimar considerando los nacimientos de nuevos individuos o el crecimiento de los que ya existen en un tiempo y por último la distribución espacial del recurso para entender la dinámica de extracción en el área (Cunningham 2001, Wong et al. 2001).

En este sentido, para la determinación de la oferta natural de Damagua en las comunidades Embera de las localidades de estudio, se establecieron tres categorías de desarrollo y cuatro clases diamétricas para los árboles. A partir de esta información, de los datos poblacionales y las prácticas tradicionales de aprovechamiento observadas en campo, se logró determinar que la oferta total de fibra de Damagua equivale a 229 árboles por 1.5 ha $(78,58$ y 93 para Agua Clara, Bella Luz y Londoño, respectivamente por 0.5 ha cada una), que corresponden a la cantidad de individuos aprovechables encontrados en las áreas muestreadas, es decir la sumatoria de los juveniles de clase diamétrica II $(10 \mathrm{~cm}$ y $20 \mathrm{~cm})$. Lo anterior equivale a 1145 sabanas de fibra obtenidas en 1.5 ha si se tiene en cuenta que, por cada árbol se elaboran hasta 5 sábanas. Las dimensiones de cada sábana de fibra de Damagua son $0.80 \mathrm{~m}$ de ancho y $1.20 \mathrm{~m}$ de largo, lo que equivale a 0.96 $\mathrm{m}^{2}$, es decir, que la oferta de la zona de muestreo alcanza una obtención de $1099 \mathrm{~m}^{2}$ en $1.5 \mathrm{ha}$. La oferta producida por cada localidad se presenta en la Tabla 1.

Para el caso de la estimación de la demanda, se tiene en cuenta que DAMAGUARTE, adquiere la materia prima cada cuatro meses, con una frecuencia anual de 3 veces, recibiendo máximo 3 bultos por vez. Cada bulto consta de 32 sábanas, luego la demanda anual es de 9 bultos que equivalen a 288 sábanas y $276 \mathrm{~m}^{2}$ de fibra. Lo anterior demanda un corte de 58 árboles por año en la zona de estudio.

Con la información de la oferta natural del recurso, obtenida a partir de las densidades aprovechables y el análisis de la productividad de la especie, se puede realizar una comparación con las cantidades demandadas, cuyos resultados son la base para del diseño de alternativas de extracción sostenible propuestas en el presente documento, las cuales se formularon de acuerdo con el contexto biológico y socioeconómico en el que se enmarca la explotación del recurso en las localidades de Bella Luz, Agua Clara y Londoño. Asimismo esta información permite determinar la oferta total de la

Tabla 1

Oferta de fibra de Damagua por localidad

\begin{tabular}{|c|c|c|c|c|}
\hline \multirow[t]{2}{*}{ Localidad } & \multicolumn{2}{|l|}{ Oferta } & \multicolumn{2}{|c|}{ Número de $\mathbf{m}^{2}$} \\
\hline & Árboles/Área muestreada & Árboles/hectárea & de sábanas/hectárea & de fibra/hectárea \\
\hline Agua Clara & 78 & 156 & 780 & 748 \\
\hline Bella Luz & 58 & 116 & 580 & 556 \\
\hline Londoño & 93 & 186 & 930 & 892 \\
\hline
\end{tabular}


Bioetnia Volumen 9 No 1 (enero-junio), 2012

Tabla 2

Resumen de oferta del recurso de Damagua en las localidades de estudio

\begin{tabular}{|c|c|c|c|c|}
\hline \multirow[t]{2}{*}{ Oferta total del recurso } & \multicolumn{2}{|c|}{ Oferta } & \multicolumn{2}{|c|}{ Oferta remanente } \\
\hline & aprovechable & aprovechada & del total & de la aprovechable \\
\hline 386 árboles/1.5ha & 229 árboles/1.5ha & 58 árboles & 157 árboles/1.5ha & 171 árboles/1.5 ha \\
\hline
\end{tabular}

zona, la oferta aprovechable y la oferta remanente de acuerdo con las cantidades actuales demandadas (Tabla 2).

El panorama expresado por las poblaciones natural de Damagua, la superioridad de la oferta frente a la demanda se convierten en elementos diagnósticos del buen estado de las poblaciones de Damagua en estas comunidades; al mismo tiempo demuestran que los indígenas pobladores han adoptado técnicas ancestrales que les permiten aprovechar el recurso en las cantidades justas y de forma permanente lo que se convierte en un elemento facilitador para el diseño de herramientas de manejo sostenible de los recursos del bosque.

\section{Conclusiones}

Es notable la fuerte y estrecha relación existente entre los indígenas Emberas habitantes del río Amporá y la oferta de fibras que los bosques producen; el uso de este recurso está asociado con las técnicas tradicionales de aprovechamiento que integran diversas actividades relacionadas con el proceso de identificación, selección y extracción de la fibra, técnicas que permiten la dedicación de un grupo especial a esta actividad, que se encarga de la enseñanza que se trasmite de generación en generación; esta enseñanza tiene un eje cultural, en el cual el fundamento principal es la buena utilización del recurso para mantenerlo y poder perpetuarlo.

La respuesta de las poblaciones de Damagua al uso, permite establecer el equilibrio existente, pues sus abundancias advierten su buena salud, esta podría ser explicada por los siguientes factores:

a. La forma y técnicas que aplican los indígenas para el aprovechamiento de la fibra, donde los diámetros menores de $10 \mathrm{~cm}$ y mayores de $20 \mathrm{~cm}$ no son utilizados, situación que les permite conservar o proteger los individuos adultos para que mantengan el aporte reproductivo a las poblaciones y a los infantes para que aporten al reclutamiento de la fase juvenil.

b. Los volúmenes de extracción ( 58 ind/año) no sobrepasan la capacidad de carga (229 árboles/1.5ha), permitiendo que la oferta se mantenga y que los juveniles que son el objeto de extracción puedan hacer su aporte en el reclutamiento para el incremento de los adultos reproductores.

c. El estado de conservación de la estructura del bosque de galería y de rivera, las poblaciones sanas de mamíferos y roedores y la figura de uso restringido (zona protectora productora) que tiene el área por parte de CODECHOCO, permite la conectividad y movilidad de especies, promoviendo que procesos como dispersión de semillas, puedan ser más efectivos, debido a que se facilita la movilización y permanencia de la fauna al interior del bosque, lo cual se refleja directamente en la abundancia, colonización y distribución natural efectiva de las poblaciones de esta especie.

Las existencias de esta especie, según esta investigación, también se ve favorecidas por la baja demanda del recurso, situación que fue corroborada al determinar una cadena productiva simple constituida por dos elementos principales: los extractores (indígena Emberas) y los artesanos transformadores (DAMAGUARTE); este modelo disminuye la presión debido a que los primeros solo extraen lo que los segundos necesitan. Estos últimos aprovechan y reciclan los residuos de la fibra optimizando el uso, además demandan bajas cantidades de la fibra, aun cuando su mercado es a diferentes escalas.

Vale la pena resaltar la voluntad de los involucrados en el buen aprovechamiento de este recurso, para el diseño de instrumentos de manejo que permitan certificar el uso amigable del recurso, de forma tal que tanto extractores como artesanos puedan acceder a los mercados internacionales, ya que por no tener dicho permiso se disminuye la competitividad y la opción de alcanzar un mercado rentable que favorecería la consolidación de una industria a partir del buen uso de los recursos naturales que ofertan nuestros ecosistemas.

Es importante advertir que existen grandes vacios de información sobre esta especie y que este estudio proporciona datos básicos y significativos sobre las poblaciones, la oferta natural, la demanda y el uso, elementos principales para la definición y elaboración de herramientas que integren la investigación, la participación comunitaria y la bioprospección de un recurso importante que favorece el bienestar de la cadena productiva, que involucra diferentes actores locales y que tiene sus inicios en el bosque de propiedad colectiva.

\section{Literatura citada}

Corporación Autónoma para el Desarrollo Sostenible del Chocó. 2010. Plan de ordenación forestal de la parte alta del río Baudó. Informe final. Quibdó: CODECHOCO. $231 \mathrm{pp}$. 
Conservación, oferta natural y estado actual de Poulsenia armata. G. Ramírez-Moreno et al.

Cunningham, A. B. 2001. Etnobotánica aplicada. Pueblos, uso de plantas silvestres y conservación. Montevideo: WWF, UNESCO, Royal Botanic Gardens Kew. p. 310.

Forero, E., A. H. Gentry. 1989. Lista anotada de las plantas del departamento del Chocó, Colombia. Bogotá: Instituto de Ciencias Naturales. Museo de Historia Natural. Biblioteca José Jerónimo Triana \# 10. Universidad Natural de Colombia. 142 pp.

Gentry, A. H. 1982. Patterns of neotropical plant diversity. Evol Biol. 15: 1-84

Macia, M., H. Romero, R. Valencia. 2001. Patrones de uso en un bosque primario de la Amazonía ecuatoriana: comparación entre dos comunidades Huaorani. En: Duivenvoorden, J., H. Balslev, J. Cavelier, C. Grandez,
H. Tuomisto, R. Valencia (eds.) Evaluación de recursos vegetales no maderables en la Amazonía noroccidental. Amsterdam: IBED, Universiteit van Ámsterdam. p. 225-50.

Pino, N., Mena, A., Valois, H., Rentería, E., Cuesta, J., A. Castro. 2001. Categorías de uso de las especies presentes en el Herbario "CHOCÓ» de la Universidad Tecnológica del Chocó. Disponible en: http:// www.botanica alb.org/Publicaciones/Otros/9Etnobot.pdf

Wong, J., K. Thornber, N. Baker. 2001. Productos forestales no madereros 13. Evaluación de los recursos de productos forestales no madereros: experiencia y principios biométricos. Roma: Organización de las Naciones Unidas para la Agricultura y la Alimentación (FAO). 124 pp. 